\title{
Introducing V-Line as a New Strategy to Choose Surgical Corridor in Oblique Lumbar Interbody Fusion at the L5- S1 Segment
}

\author{
Wei Zhang $\mathbb{D}^{\text {, Xing }}$ Du $(\mathbb{D}$, Yong Zhu, Wei Luo, Ben Wang, Guanyin Jiang, \\ and Yunsheng $\mathrm{Ou}$ \\ Department of Orthopedics, The First Affiliated Hospital of Chongqing Medical University, YouYi Road 1\#, YuZhong District, \\ Chongqing 400016, China \\ Correspondence should be addressed to Yunsheng Ou; ouyunsheng2001@163.com
}

Received 7 January 2021; Revised 23 March 2021; Accepted 5 April 2021; Published 22 April 2021

Academic Editor: Dong Pan

Copyright (c) 2021 Wei Zhang et al. This is an open access article distributed under the Creative Commons Attribution License, which permits unrestricted use, distribution, and reproduction in any medium, provided the original work is properly cited.

Purpose. A retrospective imaging study assessing the availability of oblique lumbar interbody fusion at the level of L5-S1 (OLIF51) and to choose ideal surgical corridor in OLIF51 by introducing V-line. Methods. The axial views through the center of L5-S1 disc were reviewed. We adopt $18 \mathrm{~mm}$ as the width of the simulated surgical corridor. The midline of the surgical corridor is at the center of L5-S1 disc. According to the traction distance of the left iliac vein (LCIV) and psoas major (PM), we defined all the subjects as V (+) (traction-difficultly LCIV), V (-) (traction-friendly LCIV), P (+) (traction-difficultly PM), and P (-) (traction-friendly PM). Vline was defined as a straight line dividing equally the simulated surgical corridor. All cases were divided into 2 groups: The V-line (+) group, more than half of the LCIV region, is located in the ventral part of V-line; the V-line (-) group, more than half of the LCIV region, is located in the dorsal part of V-line. Multiple variables regressive analysis was conducted to analyze the independent risk factors of V-line (+). Results. V-line $(+)$ was found in $36(38.7 \%)$ patients and V-line (-) in $57(61.3 \%)$. Incidence of $\mathrm{V}(+)$ and $\mathrm{P}(+)$ was $35.4 \%(33 / 93)$ and $30.1 \%(28 / 93)$, respectively. $16.1 \%(15 / 93)$ subjects processed $\mathrm{V}(+)$ and $\mathrm{P}$ $(+)$ at the same time. The independent risk factor of $\mathrm{V}$-line $(+)$ were gender of male $(P=0.034, \mathrm{OR}: 12.152)$ and medial position of LCIV $(P<0.001$, OR: 265.085). High iliac crest was a significant independent protective factor $(P=0.001,0 R$ : 0.750$)$. Conclusions. Most patients were suitable for OLIF51. V-line could assess the injury risk of LCIV. For patients who are V-line (+), mainly among males having the LCIV near the midline or the iliac crest relatively low, a surgical corridor external to the LCIV should be taken into consideration.

\section{Introduction}

The oblique interbody fusion (OLIF), as a new type of minimally invasive technique, has good applicability at L2-L5. It has a wide range of indications including lumbar degenerative disease, spinal deformities, trauma, infections, and neoplasms [1]. This procedure has many advantages in comparison with traditional spinal posterior approach surgery. As with ALIF and LLIF, it also avoids iatrogenic injury to the paraspinal musculature and disruption of spinal canal [2]. Besides, this surgical technique allows access to the anterolateral margin of the vertebral body between the psoas major and abdominal aorta, thus reducing the lumbar plexus injury during LLIF and abdominal large vessel injury during ALIF [3]. However, its application is often impeded for severe canal stenosis, large disc herniation, or concomitant ruptured disc herniation by its characteristics of indirect decompression. Therefore, OLIF should be carefully chosen in clinical indications [4-6].

There were a few cases using OLIF of L5-S1 in recent literatures; however, it is still quite difficult to perform OLIF51 because of the risks associated with stretch of the iliac vessels and the presence of the iliac wing disturbing insertion of the cage. Silvestre et al. [7] reported 179 patients using miniopen 
anterior retroperitoneal lumbar interbody fusion concluding that it is a safe approach for accessing the L2-L5 and advised using another approach for the L5-S1 due to the danger of injuring the iliac vessels.

Several studies have evaluated the size of OLIF51 surgical corridor. Capellades et al. [8] confirmed the great anatomic variability of vascular structures in the lumbosacral area, and the window is really small in $18.05 \%$ of the study population because of the venous structures overlapping the L5-S1 disc. To our knowledge, there is no study to analyze the anatomic structure at L5-S1 combined with OLIF surgical corridor, as the oblique operation channel will change many anatomical parameters.

The purpose of this study is to simulate the OLIF operation process at the L5-S1 level and then obtain anatomical data through CT image analysis, thus providing a way to evaluate the feasibility of OLIF51 and to minimize vascular complications in the preoperative planning stage.

\section{Methods and Methods}

2.1. Subject Characteristics. 120 consecutive patients undergoing lumbar CT examination at our hospital's Department of Radiology from 1 October 2019 to 31 December 2019 were reviewed. All subjects had a clear thin-slice CT scan. Patients who had abdominal vascular abnormalities or diseases (i.e., abdominal aortic aneurysms, Budd-Chiari syndrome, abdominal aortic dissection, and iliac artery occlusion), spinal deformity from any cause, lumbar spondylolisthesis, transitional anatomy (i.e., sacralisation of L5, or lumbarisation of S1), lumbar fracture, or a surgical history on lumbar or retroperitoneum were excluded. The CT images were obtained via PACS (Picture Archiving and Communication System) with the patient in supine position. All the radiological measurements were measured and recorded by two independent researchers, and the average value was taken as the final result. This study was approved by the institutional review board following the declaration of Helsinki principles, and informed consent was obtained from all individual participants. The whole research is being reported in line with the STROCSS criteria [9].

2.2. Surgical Simulation. The width of the OLIF cage is $18 \mathrm{~mm}$ (Medtronic, Inc, Minneapolis, Minnesota). As the actual operative window for OLIF is not less than the width of a cage, the author adopts $18 \mathrm{~mm}$ as the width of surgical corridor to simulate the operation process. The midline of surgical corridor is at the center of the L5-S1 disc, and the dorsal margin of surgical corridor is close to the left iliac crest. We assumed that the cage is inserted obliquely with the midline going through the center of the L5-S1 disc (Figure 1).

\subsection{Evaluation of Vascular Parameters}

2.3.1. The Left Iliac Vein Position (VP). On the axial plane of the L5-S1 disc, the area between the median line and the left edge of the intervertebral disc was equally divided into three zones. Patients were classified into three groups according to

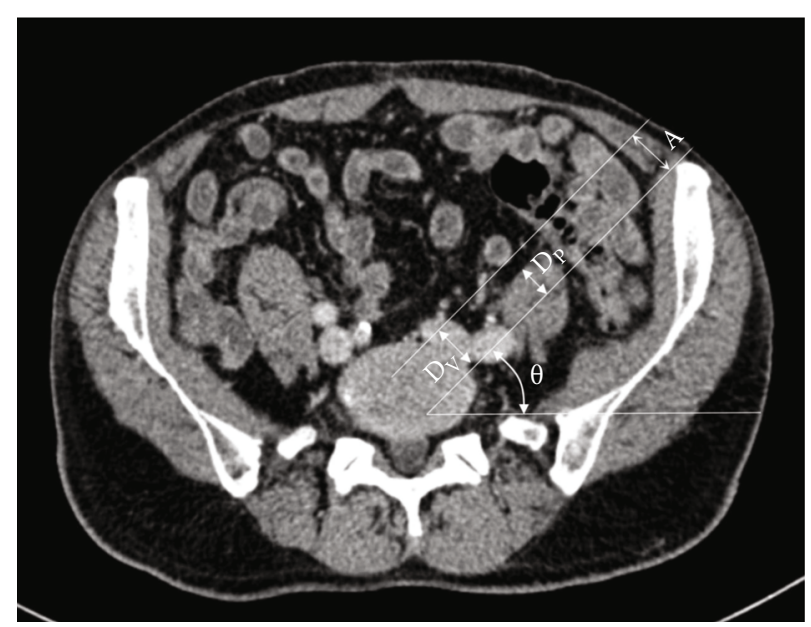

FIGURE 1: Illustration showing the simulated surgical corridor. The midline of surgical corridor is at the center of the L5-S1 disc and the dorsal margin of surgical corridor is close to left iliac crest. Distance $A$ is the diameter of the surgical corridor $(18 \mathrm{~mm}) . D_{V}$ is the traction distance of the left iliac vein. $D_{P}$ is the traction distance of the psoas major. $\theta$ is the tilt angle of surgical corridor.

their right edge of the left iliac vein: lateral, intermediate, and medial (Figure 2).

2.3.2. The Iliocaval Junction Position (JP). We used the classification established by Capellades et al. [8]. A percentage of the distance between the inferior surface of the iliocaval junction and the center of the L4-L5 disc as well as the distance between the center of the L4-L5 disc and the center of the L5-S1 disc was calculated. Patients were classified into four groups according to their junction position: very high (iliocaval junction position less than $-33.3 \%$ ), high (iliocaval junction position between $-33.3 \%$ and $33.3 \%$ ), low (iliocaval junction position between $33.4 \%$ and $66.6 \%$ ), or very low (iliocaval junction position greater than 66.7\%) (Figure 3).

2.3.3. The $V$-Line. In the present study, we proposed a new index that can evaluate the traction distance of iliac vessels. We named this index the V-line. The "V" stands for "vessel" or "vein." We defined V-line as a straight line dividing equally the surgical corridor which we simulated before. According to the $\mathrm{V}$-line, all cases were divided into 2 groups: $\mathrm{V}$-line (+) group and $\mathrm{V}$-line (-) group. In the $\mathrm{V}$-line $(+)$ group, more than half of the left iliac vein region is located in the ventral part of $\mathrm{V}$-line, so the corridor external to the left iliac vessels leads to less traction. In the V-line (-) group, more than half of the left iliac vein region is located in the dorsal part of $\mathrm{V}$-line, which indicates that a surgical corridor between the bifurcations of the iliac vessels is more optimal (Figure 4).

2.4. Quantitative Measurements. All the following parameters were analyzed and recorded in an axial plane of the center of the L5-S1 disc.

2.4.1. L5-S1 Disk Size. The anteroposterior diameter is defined as the maximal distance of the anterior and posterior border of the intervertebral disk; the left-right diameter is 


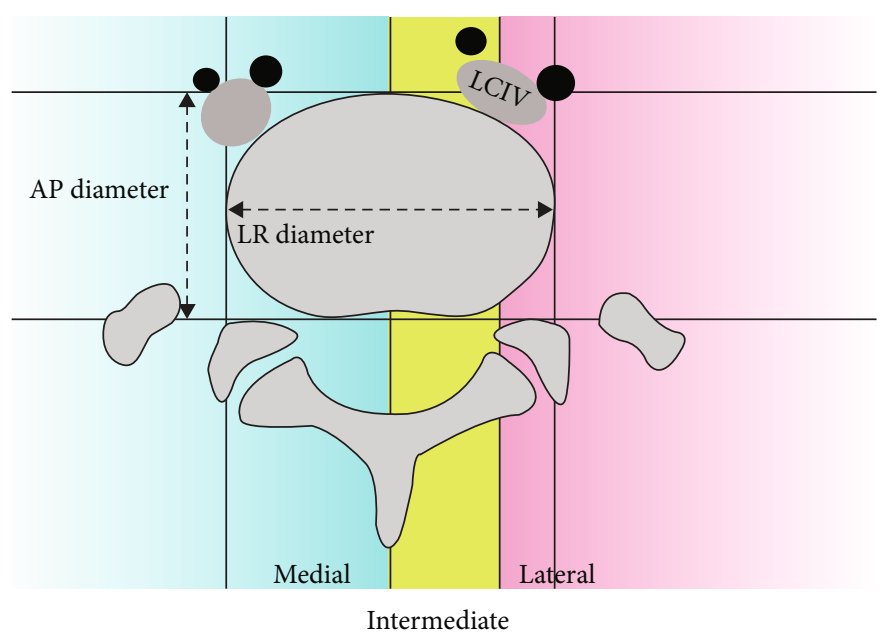

FIGURE 2: Diagram showing the anteroposterior diameter, the left-right diameter of L5-S1 disk, and the proposed classification for left common iliac vein positions. As shown in the figure, the left common iliac vein is classified into intermediate group.

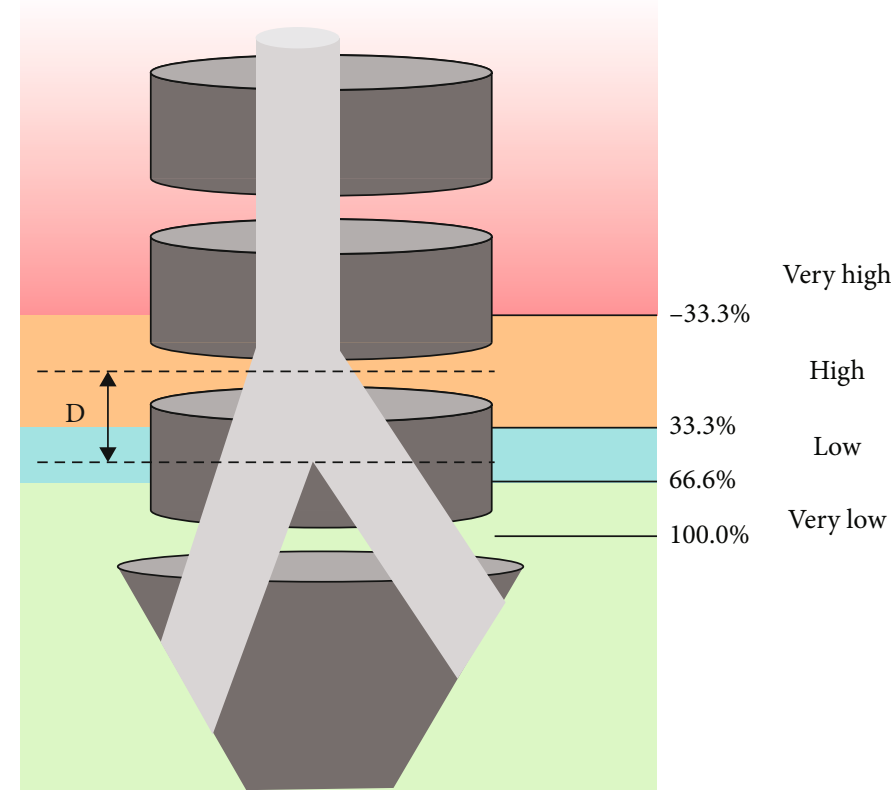

FIGURE 3: Diagram showing the proposed classification for iliocaval junction positions. $D$ is the distance between the inferior surface of the iliocaval junction and the center of the L4-L5 disc.

defined as the maximal distance of the left and right border of the intervertebral disk (Figure 2).

2.4.2. Tilt Angle of Surgical Corridor. It is defined as the angle between the surgical corridor and the horizontal line. This parameter simulated the cage implantation angle when a patient was placed in the right lateral decubitus position (Figure 1).

2.4.3. Traction Distance of the Left Iliac Vein. It is defined as the minimum distance to retract the iliac vein out of the surgical corridor (Figure 1). The LCIV that needs to be stretched more than $9 \mathrm{~mm}$ were defined as $\mathrm{V}(+)$ (traction-difficultly LCIV) and that less than $9 \mathrm{~mm}$ as $\mathrm{V}(-)$ (traction-friendly LCIV).
2.4.4. Traction Distance of the Psoas Major. It is defined as the minimum distance to retract psoas major out of the surgical corridor. In all cases, psoas major was retracted dorsally (Figure 1). The psoas was defined as $\mathrm{P}(+)$ (traction-difficultly $\mathrm{PM}$ ) and $\mathrm{P}(-)$ (traction-friendly PM) in the same way as LCIV.

2.5. Statistical Analysis. Continuous variables were presented as mean \pm SD. $\chi^{2}$ analysis was used to find a statistical difference in the left iliac vein position (VP) and the iliocaval junction position (JP) between men and women. Univariate analysis for all risk factors of $\mathrm{V}$-line $(+)$ was conducted using the 2-tailed independent Student $t$-tests for continuous variables and $\chi^{2}$ or Fisher exact test for categorical variables. A multivariate logistic regression was conducted to find 


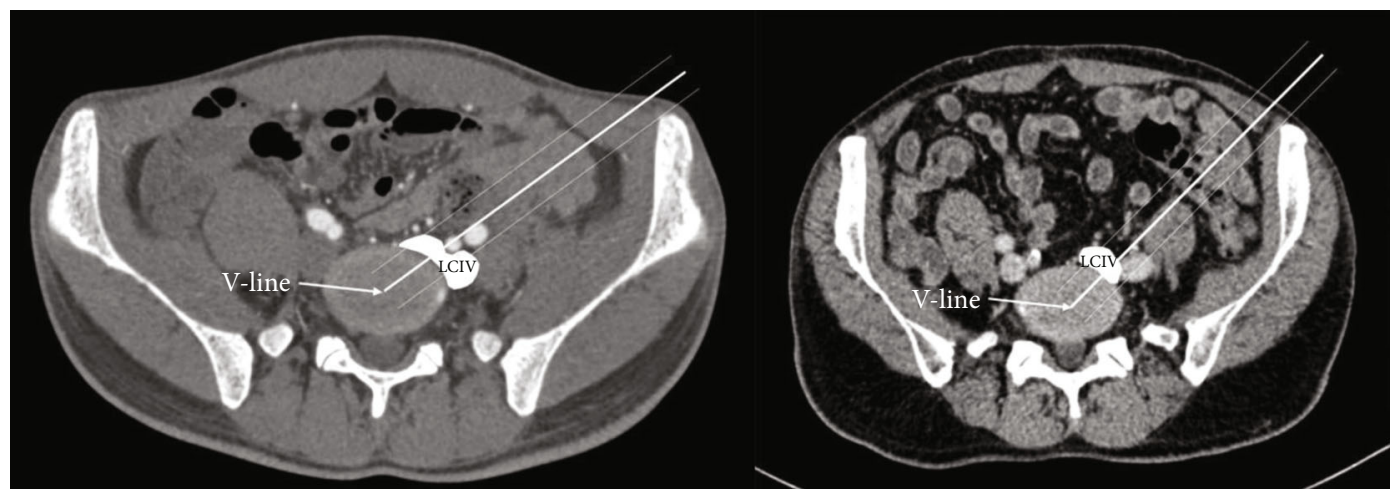

FIGURE 4: The diameter of the OLIF surgical corridor is $18 \mathrm{~mm}$. The midline of the surgical corridor is at the center of the L5-S1 disc. Left, the $\mathrm{V}$-line (-) group, more than half of the left iliac vein region is located in the dorsal part of V-line. In this situation, we could retract the LCIV dorsally. Right, the V-line (+) group, more than half of the left iliac vein region is located in ventral part of V-line. In this situation, we could retract the LCIV ventrally.

independent risk factors for $\mathrm{V}$-line $(+)$. Risk factors for V-line (+) with $P<0.15$ by univariate analysis were included in the model. Using a forward (LR), variables with a $P<0.10$ remained in the final model, with significant variables having $P<0.05$. Odds ratios (OR) and $95 \%$ confidence intervals were calculated for all variables in the model. Statistical analysis was performed using SPSS 20.0 (IBM Corporation, Armonk, New York, USA).

\section{Results}

We accessed 93 CT data in this paper, consisting of 49 men and 44 women with a mean age of $55.80 \pm 15.94$ years. A total of 27 subjects were excluded-among them 10 with transitional anatomy, 7 with huge abdominal neoplasms, 5 with spinal deformity, 4 with surgical history on lumbar or retroperitoneum, and 1 with huge lumbosacral osteophyte. The subjects' other quantitative measurements are summarized in Table 1.

According to the VP classification, 35 patients (37.6\%) were grouped in the lateral group, 33 patients $(35.5 \%)$ in the intermediate group, and 25 patients $(26.9 \%)$ in the medial group. Statistically significant difference was found between gender and the left iliac vein position. Males displayed a more medial position of LCIV.

According to the JP classification, 3 patients (3.2\%) were grouped in the very high group, 39 patients $(41.9 \%)$ in the high group, 38 patients $(40.9 \%)$ in the low group, and 13 patients $(14.0 \%)$ in the very low group. No statistically significant differences were found between gender and the iliocaval junction position (Table 2).

To evaluate precisely the ease of surgical exposure of OLIF51, the study population was classified into 4 configurations by combining traction distance of LCIV and psoas major. According to the four-configuration classification, 47 patients $(50.5 \%)$ were included in $\mathrm{P}(-) \mathrm{V}(-)$ group, 18 (19.4\%) in $\mathrm{P}(-) \mathrm{V}(+)$ group, $13(14.0 \%)$ in $\mathrm{P}(+) \mathrm{V}(-)$ group, and $15(16.1 \%)$ in $\mathrm{P}(+) \mathrm{V}(+)$ group. The $\mathrm{P}(+) \mathrm{V}(+)$ group is considered not suitable for OLIF51 due to hard exposure. There were $61.3 \%(57 / 93)$ of the subjects that were defined as V-line (-) and 38.7\% (36/93) as V-line (+) (Table 3).
Chi-square analysis or Student $t$-tests was used to compare gender, age, L5-S1 disk size, tilt angle of surgical corridor, sacral slope, the left iliac vein position (VP), and the iliocaval junction position (JP) in relation to the $\mathrm{V}$-line. Differences were observed in tilt angle of surgical corridor, sacral slope, left iliac vein position (VP), and iliocaval junction position (JP); they were all statistically significant (tilt angle of surgical corridor, $P=0.001$; sacral slope, $P=0.002$; left iliac vein position, $P<0.001$; iliocaval junction position, $P<0.001)$. The gender, age of the patient, and the L5-S1 disk size will not significantly influence the $\mathrm{V}$-line (Table 1 ).

The multivariate analysis identified gender of male $(P=0.034$, odds ratio [OR]: 12.152) and medial position of LCIV $(P<0.001$, odds ratio [OR]: 265.085) as significant independent risk factors for $\mathrm{V}$-line $(+)$, while high iliac crest was a significant independent protective factor $(P=0.001$, OR: 0.750) (Table 4).

\section{Discussion}

4.1. Application of OLIF51. OLIF51 is considered as minimally invasive ALIF through the oblique corridor in the lateral position [10]. It keeps the advantages of traditional ALIF with direct and extensive exposure of the intervertebral disc and avoidance of neural and muscular injury compared with the posterior approach. As a new surgical technique, OLIF51 could be described as laterally positioned ALIF, but the latter was superior to the former in many aspects $[3,11$, 12]. First, it can be extended to upper levels in a single position with less mobilization of the great vessels, especially for ALIF at L4-5. Secondly, it can avoid rectus abdominis muscle injury, as well as minimize the mobilization of the peritoneal content [3]. Moreover, OLIF51 is advantageous in obese patients because gravity pulls the visceral fat away from the spine [13]. Even so, OLIF at L5-S1 is still difficult because of the risks associated with mobilization of the vessels and the presence of the iliac wing.

Many literatures have proved the practicability of OLIF51 [3, 10, 14-18]. Silvestre et al. [7] first reported OLIF51 through a retroperitoneal approach was performed successfully in 6 patients, but one patient had to be aborted 
TABLE 1: Univariate analysis of risk factors for V-line (+) during OLIF51.

\begin{tabular}{|c|c|c|c|c|}
\hline Risk factors & Patients with V-line $(+), n=36$ & Patients with V-line (-), $n=57$ & $\chi^{2} / t$ & $P$ \\
\hline \multicolumn{5}{|l|}{ Sex } \\
\hline Female & 14 & 30 & \multirow{2}{*}{1.672} & \multirow{2}{*}{0.196} \\
\hline Male & 22 & 27 & & \\
\hline Age & $57.39 \pm 14.07$ & $54.79 \pm 17.06$ & -0.764 & 0.447 \\
\hline L5-S1 disk AP diameter & $37.66 \pm 3.44$ & $37.48 \pm 3.80$ & -0.229 & 0.820 \\
\hline L5-S1 disk left-right diameter & $55.95 \pm 6.49$ & $54.04 \pm 5.21$ & -1.564 & 0.121 \\
\hline Tilt angle of surgical corridor & $27.15 \pm 10.20$ & $34.05 \pm 6.72$ & 3.595 & $0.001^{*}$ \\
\hline Sacral slope & $35.42 \pm 5.34$ & $39.44 \pm 6.02$ & 3.269 & $0.002^{*}$ \\
\hline Low iliac crest $^{\#}$ & 22 & 21 & \multirow{2}{*}{5.228} & \multirow{2}{*}{$0.022 *$} \\
\hline High iliac crest & 14 & 36 & & \\
\hline \multicolumn{5}{|l|}{ The left iliac vein position (VP) } \\
\hline Medial & 22 & 3 & \multirow{3}{*}{44.570} & \multirow{3}{*}{$<0.001^{*}$} \\
\hline Intermediate & 13 & 20 & & \\
\hline Lateral & 1 & 34 & & \\
\hline \multicolumn{5}{|l|}{ The iliocaval junction position (JP) } \\
\hline Very high & 8 & 31 & \multirow{4}{*}{23.263} & \multirow{4}{*}{$<0.001^{*}$} \\
\hline High & 16 & 22 & & \\
\hline Low & 0 & 3 & & \\
\hline Very low & 12 & 1 & & \\
\hline
\end{tabular}

${ }^{*} P<0.05$, , subjects were divided into low iliac crest group and high iliac crest group according to the average value of the tilt angle of surgical corridor.

TABLE 2: Gender differences of the left iliac vein position (VP) and the iliocaval junction position (JP).

\begin{tabular}{lccc}
\hline The left iliac vein position (VP) & \multicolumn{1}{c}{ Female } & Male \\
\hline Medial & $25(26.9 \%)$ & $5(5.4 \%)$ & $20(21.5 \%)$ \\
Intermediate & $33(35.5 \%)$ & $15(16.1 \%)$ & $18(19.4 \%)$ \\
Lateral & $35(37.6 \%)$ & $24(25.8 \%)$ & $11(11.8 \%)$ \\
The iliocaval junction position (JP) & & & $1(1.1 \%)$ \\
Very high & $3(3.2 \%)$ & $2(2.2 \%)$ & $18(19.4 \%)$ \\
High & $39(41.9 \%)$ & $21(22.6 \%)$ & $21(22.6 \%)$ \\
Low & $38(40.9 \%)$ & $17(18.3 \%)$ & $9(9.7 \%)$ \\
Very low & $13(14.0 \%)$ & $4(4.3 \%)$ & 0.449 \\
\hline
\end{tabular}

TABLE 3: V-line and four-configuration classification system.

\begin{tabular}{|c|c|c|c|c|c|}
\hline & $\mathrm{P}(-) \mathrm{V}(-)$ & $\mathrm{P}(-) \mathrm{V}(+)$ & $\mathrm{P}(+) \mathrm{V}(-)$ & $\mathrm{P}(+) \mathrm{V}(+)$ & Total \\
\hline V-line (-) & $25(26.9 \%)$ & $8(8.6 \%)$ & $9(9.7 \%)$ & $15(16.1 \%)$ & $57(61.3 \%)$ \\
\hline V-line (+) & $22(23.7 \%)$ & $10(10.8 \%)$ & $4(4.3 \%)$ & $0(0.0 \%)$ & $36(38.7 \%)$ \\
\hline Total & 47 (50.5\%) & $18(19.4 \%)$ & $13(14.0 \%)$ & $15(16.1 \%)$ & $93(100.0 \%)$ \\
\hline
\end{tabular}

and switched to another operation. Woods et al. [3] retrospectively evaluated 137 patients who underwent the OLIF procedure, in which 10 patients who underwent OLIF51 only, and 84 patients who underwent OLIF25 combined with OLIF51; then, the author draws a conclusion that OLIF is a safe procedure at L1-5 as well as L5-S1. Mun et al. [19] retrospectively reviewed and compared 74 patients who underwent OLIF51 and 74 who underwent TLIF51 and concluded that OLIF51 was more effective for indirect decompression of foraminal stenosis, providing strong mechanical support. Zairi et al. [14] also showed the feasibility of accessing the L5-S1 level through the mini-open retroperitoneal approach without the need for ALIF.

4.2. OLIF51 Surgical Window. Similar to ALIF L5-S1, OLIF51 typically involves the corridor under the bifurcation of the great abdominal vessels [3]. Tribus and Belanger [20] performed a cadaveric study in 35 cadavers to examine the size 
TABLE 4: Multivariate analysis of risk factors for V-line (+) during OLIF51.

\begin{tabular}{lccc}
\hline Risk factor & Odds ratio & $95 \%$ confidence interval & $P$ \\
\hline Sex & 12.152 & $1.208-122.276$ & 0.034 \\
The left iliac vein position (VP) & 265.085 & $16.629-4225.839$ & $0.632-0.890$ \\
Tilt angle of surgical corridor & 0.750 & - & 0.001 \\
Age & - & - & 0.001 \\
L5-S1 disk AP diameter & - & - & 0.712 \\
L5-S1 disk left-right diameter & - & - & 0.970 \\
Sacral slope & - & 0.696 \\
The iliocaval junction position (JP) & - & 0.364 \\
\hline
\end{tabular}

of the central window; the mean distance from the bifurcation to the top of the L5-S1 disc was $18 \mathrm{~mm}$, and the mean width between the left common iliac vein and the right common iliac artery averaged $33.5 \mathrm{~mm}$. In 2014, Davis et al. [21] defined the OLIF51 corridor through 2 measurements. The lateral window was the distance transversely from the midsagittal line of the inferior endplate of L- 5 to the medial border of the left common iliac vessel, while the vertical window to the first vascular structure that crosses midline. The author measured the corridor diameters in 20 cadavers in a static state and with mild traction of the psoas and found that the L5-S1 corridor mean distance was $14.75 \mathrm{~mm}$ transversely and $23.85 \mathrm{~mm}$ vertically. Davis et al. concluded that the L5$\mathrm{S} 1$ disc space can be accessed from an oblique angle. Oblique access to L5-S1 has also been studied through radiological measurement. Molinares et al. [22] measured that the L5-S1 corridor mean distance was $10 \mathrm{~mm}$ between midline and left common iliac vessel and $10.13 \mathrm{~mm}$ from the first midline vessel to the inferior endplate of L-5. The author found access to the L5-S1 disc established in 69\% of the MR images analyzed. However, all these studies were just simple measurements of natural anatomic spaces, without considering the influence of oblique corridor on anatomical parameter. In addition, at the L5-S1 level, the psoas muscle is often lifted off the spine to leave the pelvis, which could also obstruct the surgical corridor. Our aim was to simulate the OLIF operation process at the L5-S1 level and then evaluate the feasibility of OLIF51 based on CT images. Similar studies have not been described in the literature.

4.3. Simulation of OLIF51. In our study, the author adopted $18 \mathrm{~mm}$ as the width of surgical corridor to simulate the operation process. We had reason to believe that tractiondifficultly structure will obstruct the OLIF working corridor, and there will be excessive stretch of these structures during the procedure. More than $1 / 3$ of the subjects $(33 / 93,35.5 \%)$ were grouped into traction-difficultly LCIV and nearly three in $10(28 / 93,30.1 \%)$ were grouped into traction-difficultly PM. Further analysis revealed that about one-sixth of the subjects $(15 / 93,16.1 \%)$ processed traction-difficultly LCIV and traction-difficultly PM at the same time. Because left common iliac vein and psoas major account for more than half of OLIF working corridor, causing difficulty in operating channel placement and increased injury risk of these structures. Therefore, we consider these subjects not suitable for
OLIF51, while the P (-) V (-) group is obviously the most ideal situation. And as for the other two groups, namely, the $\mathrm{P}(-) \mathrm{V}(+)$ group and $\mathrm{P}(+) \mathrm{V}(-)$ group, the former gives us a large operation space for psoas major and the latter for vessel. This classification could help spine surgeons make preoperative planning and guide the surgical management.

The anatomical structure of the lumbosacral segment is complex and adjacent to the important structure. Damage to LCIV is the most threatening complication associated with OLIF51 which can be very difficult to control once injured, and mobilization of these vascular structures is often a technically demanding procedure $[15,23]$. The injury to LCIV is also the most commonly complication observed in clinical practice $[8,19]$. This phenomenon is caused by the low iliocaval junction positions and the medially located LCIV, reducing the size of the operating field. According to reports in the literature, the vascular injury in the early result of OLIF was $8.6 \%$, and it increases when the L5-S1 segment is involved $[3,24]$. Therefore, careful preoperative evaluation of the vascular structure is desperately needed during OLIF at L5-S1.

In 2017, Chung et al. [18] evaluated the configuration of LCIV and its risk of mobilization during anterior approach at the L5-S1 segment. They postulated the presence of perivascular adipose tissue under the LCIV, and they categorized the LCIV into three types: type I (no requirement for mobilization), type II (easy mobilization), and type III (potentially difficult mobilization). In their study, type I LCIV configuration was found in $32(49.2 \%)$ patients, type II in 18 (27.7\%), and type III in $15(23.1 \%)$. There were $7(10.8 \%)$ patients with LCIV injury (type I, $n=0$; type II, $n=2$; type III, $n=5$ ) $(P=0.003)$. The result indicated that their LCIV classification system is valid for the evaluation of LCIV injury during its mobilization. However, we noted that there were still 2 patients of type II who had LCIV injury. In addition to improving surgical techniques, how could we further reduce the vascular injury at OLIF51?.

4.4. Assessment of $V$-Line. During the surgery, surgeons have to mobilize LCIV for surgical exposure when it obstructs the operative window on the L5-S1 disc. We could mobilize LCIV laterally choosing the central disk space between the bifurcations or stretch it medially choosing lateral disk space external to the left iliac vessels. However, which approach might reduce the risk of mobilization has not been 
comprehensively studied. Previous work on the morphological characteristics of the LCIV cannot solve the problem properly. In this thesis, a new concept, V-line, is proposed for assessing the mobilization risk of these two approaches. By introducing the concept of $\mathrm{V}$-line, we could qualitatively evaluate the extent of vascular traction of two approaches to guide surgical treatment.

In our work, there were $61.3 \%(57 / 93)$ of the subjects were defined as V-line (-). In this group, a surgical corridor between the bifurcations of the iliac vessels is more favorable which is exactly the mainstream approach in the world. One important concern with this approach is the injury to the SHP (superior hypogastric plexus), which overlies the L5-S1 disk between the bifurcations and supplies the sympathetic function for the urogenital system [25]. Consequently, damage to the SHP could result in retrograde ejaculation in male patients [26]. Careful unilateral blunt dissection of the SHP and avoidance of monopolar coagulation is recommended $[3,17]$. Besides, middle sacral vessels are also important structures during this central approach; we could simply divide these vessels by the application of bipolar cautery or vascular clips.

Naturally, the rest of the subjects $(36 / 93,38.7 \%)$ were defined as V-line (+), in which the corridor external to the left iliac vessels is superior for less stretch of the LCIV. On this condition, particular attention should be paid to the identification and handling of the iliolumbar vein (ILV) [13]. The ILV travels laterally approximately $3-4 \mathrm{~cm}$ below the bifurcation and then traverses medially along the L5 vertebral body, coursing between the obturator nerve and lumbar trunks [27]. In exposing the L5-S1 level external to the LCIV, the ILV is easily avulsed due to medial traction of LCIV. Zairi et al. had gained access to the L5-S1 level by finding and clipping the ILV before retracting the iliac artery and vein anteriorly. This study considered this a safer and feasible approach [14]. Coagulation or ligation is recommended in case the ILV is identified. We could also take gentle dissection of the fat around the L5-S1 level lateral to the iliac vessels in order to obtain better recognition of the ILV.

Recently, many studies analyzing the venous anatomy in the lumbosacral area have been reported. Whether they are useful for predicting the surgical approach of OLIF51? Multiple variables regressive analysis demonstrated that gender of male, medial position of LCIV, and high iliac crest were predictive factors of V-line, while age of the patient, L5-S1 disk size, sacral slope, and iliocaval junction position (JP) were not. As to the left iliac vein position (VP), it is found that almost all subjects of the medial group were classified into the $\mathrm{V}$-line $(+)$ group and they accounted for nearly $2 / 3$ of the V-line (+) group. In addition, the height of iliac crest is also crucial for the preoperative evaluation, there were more than half of low iliac crest group could choose an external corridor to decrease the stretch of the LCIV. However, the iliocaval junction position (JP) was not a crucial factor. A possible explanation for this is that although lower junction positions have more medial LCIV, it is not the only factor, the junction angle will affect the distribution pattern of iliac vein as well. Therefore, in the clinical practice of OLIF51, a comprehensive, detailed analysis and conventional CT exam- ination is important to minimize the vascular injury. For patients who are $\mathrm{V}$-line (+), mainly among males having the LCIV near the midline or the iliac crest relatively low, a surgical corridor external to the LCIV should be taken into consideration, and vice versa. Finally, it is worth noting that all the subjects in $\mathrm{P}(+) \mathrm{V}(+)$ group were divided into V-line (-) taking almost a quarter of the V-line (-) group, indicating potential difficulties in operating procedure.

4.5. Limitations. The present research has several limitations. First, the study object of this article is patients undergoing lumbar CT examination, whose regional anatomical character in the lumbosacral area are different from those with lumbar degeneration disease. In addition, we did not include the disc height in the measurement which might change in different pathologies and impact the result of the iliocaval junction position. The second limitation concerns the patient's position, as CT images are obtained in the supine position, whereas OLIF is performed in the right lateral decubitus position, the location and configuration of LCIV and psoas major may vary [28]. Third, all the subjects in our study were Chinese; the positive rate of $\mathrm{V}$-line might be different in other ethnic populations. Lastly, a high-quality prospective study is much needed to confirm the validity of this retrospective anatomical imaging study.

\section{Conclusions}

This study demonstrated that the majority of the patients were suitable for OLIF51 without excessive traction of the LCIV and the psoas major. There was a relatively high incidence of V-line $(+)$ in the Asian population. Among male patients having the LCIV near the midline or the iliac crest relatively low, a surgical corridor external to the LCIV should be taken into consideration to minimize the risk of vascular injury.

\section{Trial Registry Number}

The study had been registered in chictr.org.cn (UIN = ChiCTR2000038598). Full detail can be accessed via http:// www.chictr.org.cn/showprojen.aspx?proj=61903.

\section{Abbreviations \\ LCIV: Left common iliac vein \\ OLIF: Oblique lumbar interbody fusion \\ ALIF: Anterior lumbar interbody fusion \\ LLIF: Lateral lumbar interbody fusion \\ VP: Left iliac vein position \\ JP: Iliocaval junction position.}

\section{Data Availability}

The datasets used to support the findings of this study are available from the corresponding author upon request. 


\section{Ethical Approval}

This study was approved by the institutional review board following the declaration of Helsinki principles.

\section{Consent}

Informed consent was obtained from all individual participants. The whole research is being reported in line with the STROCSS criteria.

\section{Conflicts of Interest}

The authors declare that they have no competing interests.

\section{Acknowledgments}

This article has been presented as preprint according to the following link: https://www.researchsquare.com/article/rs$118245 / \mathrm{v} 1$. This work was supported by the Basic Research and Frontiers Exploration Project of Yuzhong District, Chongqing (2018114) and the Science and Technology Innovation Project for Postgraduate of Chongqing Municipal Education Commission (CYS18200). All authors gratefully acknowledge the support of the subjects who participated in the present study.

\section{References}

[1] K. Phan, M. Maharaj, Y. Assem, and R. J. Mobbs, "Review of early clinical results and complications associated with oblique lumbar interbody fusion (OLIF)," Journal of clinical neuroscience : official journal of the Neurosurgical Society of Australasia, vol. 31, pp. 23-29, 2016.

[2] J. Allain and T. Dufour, "Anterior lumbar fusion techniques: ALIF, OLIF, DLIF, LLIF, IXLIF,” Orthopaedics \& Traumatology: Surgery \& Research, vol. 106, no. 1, pp. S149-S157, 2020.

[3] K. R. Woods, J. B. Billys, and R. A. Hynes, "Technical description of oblique lateral interbody fusion at L1-L5 (OLIF25) and at L5-S1 (OLIF51) and evaluation of complication and fusion rates," The Spine Journal, vol. 17, no. 4, pp. 545-553, 2017.

[4] D. H. Heo and J. S. Kim, "Clinical and radiological outcomes of spinal endoscopic discectomy-assisted oblique lumbar interbody fusion: preliminary results," Neurosurgical Focus, vol. 43, no. 2, article E13, 2017.

[5] J. Quillo-Olvera, G. X. Lin, H. J. Jo, and J. S. Kim, "Complications on minimally invasive oblique lumbar interbody fusion at L2-L5 levels: a review of the literature and surgical strategies," Annals of Translational Medicine, vol. 6, no. 6, p. 101, 2018.

[6] D. S. Xu, C. T. Walker, J. Godzik, J. D. Turner, W. Smith, and J. S. Uribe, "Minimally invasive anterior, lateral, and oblique lumbar interbody fusion: a literature review," Annals of Translational Medicine, vol. 6, no. 6, p. 104, 2018.

[7] C. Silvestre, J. M. Mac-Thiong, R. Hilmi, and P. Roussouly, "Complications and morbidities of mini-open anterior retroperitoneal lumbar interbody fusion: oblique lumbar interbody fusion in 179 patients," Asian spine journal, vol. 6, no. 2, pp. 89-97, 2012.

[8] J. Capellades, F. Pellisé, A. Rovira, E. Grivé, S. Pedraza, and C. Villanueva, "Magnetic resonance anatomic study of iliocava junction and left iliac vein positions related to L5-S1 disc," Spine (Phila Pa 1976), vol. 25, no. 13, pp. 1695-1700, 2000.

[9] R. Agha, A. Abdall-Razak, E. Crossley, N. Dowlut, C. Iosifidis, and G. Mathew, "STROCSS 2019 Guideline: strengthening the reporting of cohort studies in surgery," International journal of surgery (London, England), vol. 72, pp. 156-165, 2019.

[10] N. Anand, A. Alayan, A. Agrawal, S. Kahwaty, E. Nomoto, and B. Khandehroo, "Analysis of spino-pelvic parameters and segmental lordosis with L5-S1 oblique lateral interbody fusion at the bottom of a long construct in circumferential minimally invasive surgical correction of adult spinal deformity," World Neurosurgery, vol. 130, pp. e1077-e1083, 2019.

[11] R. C. Sasso, J. Kenneth Burkus, and J. C. LeHuec, "Retrograde ejaculation after anterior lumbar interbody fusion: transperitoneal versus retroperitoneal exposure," Spine (Phila $\mathrm{Pa}$ 1976), vol. 28, no. 10, pp. 1023-1026, 2003.

[12] B. K. Weiner, M. Walker, and R. D. Fraser, "Vascular anatomy anterior to lumbosacral transitional vertebrae and implications for anterior lumbar interbody fusion," The Spine Journal, vol. 1, no. 6, pp. 442-444, 2001.

[13] N. S. Chung, H. D. Lee, and C. H. Jeon, "Vascular anatomy and surgical approach in oblique lateral interbody fusion at lumbosacral transitional vertebrae," Journal of orthopaedic science : official journal of the Japanese Orthopaedic Association, 2020.

[14] F. Zairi, T. P. Sunna, H. J. Westwick et al., "Mini-open oblique lumbar interbody fusion (OLIF) approach for multi-level discectomy and fusion involving L5-S1: preliminary experience," Orthopaedics \& traumatology, surgery \& research : OTSR., vol. 103, no. 2, pp. 295-299, 2017.

[15] N. S. Chung, C. H. Jeon, and H. D. Lee, "Use of an alternative surgical corridor in oblique lateral interbody fusion at the L5S1 segment: a technical report," Clinical Spine Surgery: A Spine Publication, vol. 31, no. 7, pp. 293-296, 2018.

[16] K. Kanno, S. Ohtori, S. Orita et al., "Miniopen oblique lateral L5-s1 interbody fusion: a report of 2 cases," Case reports in orthopedics, vol. 2014, Article ID 603531, 5 pages, 2014.

[17] J. S. Kim and S. B. Sharma, "How I do it? Oblique lumbar interbody fusion at L5S1(OLIF51)," Acta Neurochirurgica, vol. 161, no. 6, pp. 1079-1083, 2019.

[18] N. S. Chung, C. H. Jeon, H. D. Lee, and H. J. Kweon, "Preoperative evaluation of left common iliac vein in oblique lateral interbody fusion at L5-S1," European Spine Journal, vol. 26, no. 11, pp. 2797-2803, 2017.

[19] H. Y. Mun, M. J. Ko, Y. B. Kim, and S. W. Park, "Usefulness of oblique lateral interbody fusion at L5-S1 level compared to transforaminal lumbar interbody fusion," Journal of Korean Neurosurgical Society, vol. 63, no. 6, pp. 723-729, 2020.

[20] C. B. Tribus and T. Belanger, "The vascular anatomy anterior to the L5-S1 disk space," Spine (Phila Pa 1976), vol. 26, no. 11, pp. 1205-1208, 2001.

[21] T. T. Davis, R. A. Hynes, D. A. Fung et al., "Retroperitoneal oblique corridor to the L2-S1 intervertebral discs in the lateral position: an anatomic study," Journal of Neurosurgery. Spine, vol. 21, no. 5, pp. 785-793, 2014.

[22] D. M. Molinares, T. T. Davis, and D. A. Fung, "Retroperitoneal oblique corridor to the L2-S1 intervertebral discs: an MRI study," Journal of Neurosurgery. Spine, vol. 24, no. 2, pp. 248-255, 2016.

[23] N. A. Quraishi, M. Konig, S. J. Booker et al., “Access related complications in anterior lumbar surgery performed by spinal 
surgeons," European Spine Journal, vol. 22, no. S1, pp. 16-20, 2013.

[24] J. Garg, K. Woo, J. Hirsch, J. D. Bruffey, and R. B. Dilley, "Vascular complications of exposure for anterior lumbar interbody fusion," Journal of vascular surgery., vol. 51, no. 4, pp. 946-950, 2010.

[25] S. Eid, J. Iwanaga, J. R. Chapman, R. J. Oskouian, M. Loukas, and R. S. Tubbs, "Superior hypogastric plexus and its surgical implications during spine surgery: a review," World Neurosurgery, vol. 120, pp. 163-167, 2018.

[26] H. Tiusanen, S. Seitsalo, K. Osterman, and J. Soini, "Retrograde ejaculation after anterior interbody lumbar fusion," European Spine Journal, vol. 4, no. 6, pp. 339-342, 1995.

[27] M. Davis, S. Jenkins, S. Bordes et al., "Iliolumbar vein: anatomy and surgical importance during lateral transpsoas and oblique approaches to lumbar spine," World Neurosurgery, vol. 128, pp. e768-e772, 2019.

[28] F. Zhang, H. Xu, B. Yin et al., "Does right lateral decubitus position change retroperitoneal oblique corridor? A radiographic evaluation from L1 to L5," European Spine Journal, vol. 26, no. 3, pp. 646-650, 2017. 